\title{
A Mannose-specific Recognition Mediates the Defasciculation of Axons in the Leech CNS
}

\author{
Birgit Zipser and Robert N. Cole \\ Department of Physiology, Michigan State University, East Lansing, Michigan 48824
}

\begin{abstract}
We are studying a mannose-specific recognition mediating the projection of axons in the synaptic neuropil of the embryonic leech CNS. A functional class of neurons, the sensory afferents, can be distinguished by a mannose-containing epitope that is asparagine-linked to a $130 \mathrm{kDa}$ surface protein and is reactive with the monoclonal antibody Lan32. Sensory afferents project as a tightly fasciculated bundle through peripheral nerves but, upon arriving in the CNS, defasciculate into the synaptic neuropil. This defasciculation allows the previously bundled sensory afferents to form an arborization in the synaptic neuropil. Three lines of experimental evidence indicate that the defasciculation is mediated by the sensory afferent's mannose-containing Lan3-2 epitope. The defasciculation is inhibited (1) by blocking the Lan3-2 epitope with Lan3-2 Fab fragments, (2) by cleaving the asparagine-linked carbohydrate moieties from surface proteins with the glycosidase $\mathrm{N}$-glycanase, and (3) by competing for a putative mannose-binding protein with the neoglycoprotein mannose-BSA [albumin, p-aminophenyl $\alpha-D-$ mannopyranoside (26 mol monosaccharide/mol albumin)]. In addition to inhibiting the defasciculation, the three perturbation reagents also elicited the refasciculation of axons that had defasciculated prior to their application. These three different experimental approaches provide strong evidence that carbohydrate recognition regulates the projections of sensory afferents in the leech synaptic neuropil. Carbohydrate interactions therefore can play a major role in regulating the neuronal architecture in the CNS.
\end{abstract}

Carbohydrate recognition has long been postulated to play a role in the patterning of neuronal networks. There is increasing evidence, mostly from correlative or in vitro experiments, that the interactions between cell surface oligosaccharides and carbohydrate-binding proteins regulate different aspects of neuronal growth (reviewed in Jessell et al., 1990).

In non-neuronal systems, a combination of approaches has been used to provide direct evidence that cellular recognition involves the interactions of oligosaccharides with carbohydratebinding proteins (Ofek and Sharon, 1988; Shur, 1989; Yednock and Rosen, 1989). One experimental approach is to block carbohydrate interactions with antibodies that bind to carbohydrate antigens. Although providing a rapid method for impli-

\footnotetext{
Received Jan. 16, 1991; revised Apr. 23, 1991; accepted June 10, 1991.

This work was supported by NIH Grant NS 25117 . We thank S. Heidemann, W. J. Kopachik, M. B. Rheuben, J. Wang, and K. Zipser for discussions and critical reading of the manuscript.

Correspondence should be addressed to Birgit Zipser, Department of Physiology, 111 Giltner Hall, Michigan State University, East Lansing, MI 48824-1101.

Copyright (C) 1991 Society for Neuroscience $0270-6474 / 91 / 113471-10 \$ 05.00 / 0$
}

cating cell surface carbohydrates in cell-cell interactions, antibodies may elicit their effects by altering cell surface topology, by cross-linking cells, or by being internalized. Therefore, additional manipulations involving enzymes or soluble inhibitors are commonly used. Similarly, in the leech nervous system, we are using three types of experimental manipulations to obtain direct evidence that oligosaccharides mediate cell-cell recognition. The permeability of the cultured leech embryo to antibodies, glycosidases, and neoglycoproteins makes it possible to investigate directly the role of surface oligosaccharides in normal neuronal pattern formation.

A functional class of leech neurons, the sensory afferents, can be distinguished by their cell surface carbohydrate epitopes (Bajt et al., 1990a). The monoclonal antibody (mAb) Lan3-2 binds to a mannose-containing epitope located on the sensory afferents' $130 \mathrm{kDa}$ surface protein, the sensory afferent gp130. Normally, sensory afferent cell bodies in peripheral organs project axons as fasciculated bundles through peripheral nerves into the CNS. Upon entering the CNS, they abruptly defasciculate into the synaptic neuropil, creating a profuse arborization that precedes the stereotypic CNS pattern seen in the adult $n \approx$ uropil (Fig. 1). Exposing sensory afferents to nanomolar amounts of Lan3-2 Fab fragments had a striking region-specific effect. While sensory afferents still elongated as fasciculated bundles through peripheral nerves, they failed to defasciculate into the synaptic neuropil. Instead, they continued growing through the CNS as a fasciculated bundle. This highly altered axonal pattern resulting from the Lan3-2 perturbation provided the first direct evidence that a mannose-specific recognition regulates the growing behavior of leech sensory afferents (Zipser et al., 1989). It is possible, however, that Fab fragments binding to the mannose-containing Lan 3-2 epitope could have sterically obstructed other parts of the sensory afferent gpl 30 containing the actual domain that mediates axonal defasciculation. Therefore, we used two other experimental approaches to strengthen the evidence that a mannose-specific recognition mediates the defasciculation of sensory afferents. Here, we report that the removal of the mannose-containing chains on asparagine $(\mathrm{N})$-linked carbohydrate domains with $N$-glycanase treatment and the blocking of a putative mannose-binding protein with mannose covalently linked to BSA [mannose-BSA; albumin, $p$-aminophenyl $\alpha$-Dmannopyranoside (26 mol monosaccharide/mol albumin)] mimicked the effects obtained with the application of Lan3-2 Fab fragments. Together, the results from these three independent kinds of perturbations make a strong case that the mannose-containing Lan3-2 epitope mediates the defasciculation of sensory afferent axons.

In addition to mediating the defasciculation of sensory afferents, the Lan3-2 epitope also appears to be involved in stabi- 
lizing the arborizations of sensory afferents in the synaptic neuropil. Previously formed sensory afferent arborizations are replaced with fasciculated tracts during the three kinds of perturbation experiments. Thus, sensory afferents use their Lan3-2 epitope both to form and to maintain their stereotypic CNS pattern.

\section{Materials and Methods}

All of the experiments were performed on Hirudo medicinalis embryos. The embryos were harvested from cocoons that were collected from laying chambers kept at $25^{\circ} \mathrm{C}$, and then grown at $20^{\circ} \mathrm{C}$. Only cocoons containing between 15 and 19 embryos at the same state of maturation were used; the occasional runt among these embryos was discarded. Most experiments were performed on embryos in which sensory afferents had innervated the CNS as far posteriorly as midbody ganglia 1618 , as occurs in 10.5-d-old embryos. Other experiments were performed on embryos with sensory afferents innervating only the most anterior midhody ganglia as occurs in 9-d-old embryos. To identify the midbody ganglia, the anteriormost pair of kidneys located between midbody ganglia 1 and 2 is used as a landmark.

Culturing embryos. Each embryo was opened with a dorsal incision in its epithelial envelope to remove the yolk and to expose the germinal plate that is attached to the ventral part of the envelope. The germinal plates of embryos of a given cocoon were divided into different categories, each category containing at least three siblings. To determine the developmental stage of sensory afferent differentiation, germinal plates were fixed at the onset of culturing. The germinal plates to be cultured were washed four times with sterile tissue culture medium by passing them through four wells of a 24-well Falcon plate (Fisher Scientific). They were then stretched out by pinning their epithelial envelope on UV-sterilized Sylgard-coated culture dishes $(35 \times 10 \mathrm{~mm}$ Falcon Petri dishes, Fisher Scientific). The three to five germinal plates belonging to the same category were cultured in the same dish in $1 \mathrm{ml}$ of culture medium for $1.5 \mathrm{~d}$ at $20^{\circ} \mathrm{C}$. The standard culture medium is a defined medium consisting of Lcibovitz- 15 whose potassium concentration was adjusted to be $15 \mathrm{~mm}$, supplemented with $1 \% \mathrm{ITS}^{+}$@ $(\mathrm{Col}-$ laborative Research Inc., Bedford, MA), $10 \mathrm{nM}$ CR epidermal growth factor (Collaborative Research Inc.), $100 \mu \mathrm{M}$ vitamin C, and $0.15 \%$ glucose. In antibody perturbation studies, 10 nM Lan3-2 or Laz10-1 Fab fragments were added. mAb Lan 3-2 recognizes the sensory afferent gp130 (Zipser and McKay, 1981; Bajt et al., 1990a), and mAb Laz10-1 recognizes a muscle-specific external protein (Thorey and Zipser, 1991). In sugar perturbation studies, the following neoglycoproteins were added: mannose-BSA [ $26 \mathrm{~mol}$ of $p$-aminophenyl $\alpha$-D-mannopyranoside/ mol albumin (Sigma A-4664)], galactose-BSA (26 mol 2-amido-2-deoxy$d$-galactose/mol albumin (Sigma A-5908)], or galactosamine-BSA [19 mol $p$-aminophenyl $N$-acetyl- $\beta$-D-galactosamide/mol albumin (Sigma A-1159)]. In enzyme perturbation studies, germinal plates were grown in $0.3 \mathrm{ml}$ of culture medium in Sylgard-coated LabTek chambers (Nunc Inc., Naperville, IL) to which $1-4 \mathrm{U}$ of $N$-glycanase $(60 \mathrm{U} / \mathrm{ml}$; Genzyme Corp., Boston, MA) were added.

Immunocytochemistry. Germinal plates were washed in $50 \mathrm{~mm}$ phosphate-buffered saline (PBS) and fixed for $0.5 \mathrm{hr}$ with $4 \%$ paraformaldehyde in $0.1 \mathrm{~m}$ phosphate buffer, $\mathrm{pH}$ 7.4. Endogenous peroxidases were destroyed by immersion in methanol for $30 \mathrm{~min}$. Germinal plates were permeabilized with xylene and returned into PBS through sequential washes of $100 \%$ methanol, $95 \%$ ethanol, and $70 \%$ ethanol. The germinal plates were incubated overnight with $\mathrm{mAbs}$, followed by a $2 \mathrm{hr}$ treatment with biotinylated $\mathrm{F}\left(\mathrm{ab}^{\prime}\right)_{2}(1: 100 ;$ DAKO Corp., Santa Barbara, CA) and then a $1 \mathrm{hr}$ treatment with avidin-horseradish peroxidase (HRP) (1: 150; Vector Laboratories Inc., Burlingame, CA). Antibodies and avidinHRP were diluted in PBS containing 3\% BSA. All antibody incubation and washing steps were carried out in the presence of $2 \%$ Triton X-100. After the detergent was removed through a rinse with PBS, the germinal plates were reacted with $0.02 \% 3,3^{\prime}$-diaminobenzidine in $50 \mathrm{~mm}$ Tris buffer ( $\mathrm{pH} 7.4$ ) containing $0.015 \%$ hydrogen peroxide, rinsed with PBS, dehydrated with ethanol, cleared in xylene, and embedded in Permount. The germinal plates were photographed through a $100 \times$ Zeiss Planapo objective.

Lan3-2 ascites fluid was used at a dilution of 1:4000 to stain the sensory afferents. Sensory afferents proteins partially deglycosylated by $N$-glycanase were only weakly stained with mAb Lan 3-2. To amplify Lan 3-2 staining of sensory afferents, the staining procedure was per- formed twice as follows: germinal plates were incubated with I an 3-2 followed by HRP-conjugated goat anti-mouse IgG (1:50; Cappel, Westchester, NY) and were incubated again with Lan 3-2 followed by a second exposure to HRP-conjugated goat anti-mouse IgG. Glial processes were stained with undiluted mAb Laz6-56 hybridoma supernatant, which reacts with a $130 \mathrm{kDa}$ glycoprotein on glial processes and produces as similar staining pattern as mAb Laz6-297 (Flaster and Zipser, 1987; Cole et al., 1989).

Generation of Fab fragments. Fab fragments were prepared from Lan3-2 and Laz10-1 ascites fluid that had been ammonium sulfate precipitated, digested with mercuripapain (Worthington Biochemical Corp., Freehold, NJ), and subjected to ion exchange chromatography according to Mishell and Shiigi (1980). The concentration of Fab fragments per fraction was estimated assuming that $1 \mathrm{mg} / \mathrm{ml}$ of immunoglobulin has an absorption of 1.4 at $280 \mathrm{~nm}$. The protein concentration was also determined using a Coomassie blue binding assay (Bradford, 1976). As determined on silver-stained SDS-polyacrylamide gels, Lan3-2 and Laz 10-1 Fab fragments had molecular weights of $55 \mathrm{kDa}$. The Lan3-2 perturbation effect was elicited with Fab fragments of $0.7 \mu \mathrm{g} / \mathrm{ml}$ corresponding to $10 \mathrm{nM}$.

Measurements of sensory afferent projections by image analysis. Initial measurements of the area of sensory afferent projections were performed on drawings of micrographs (Fig. 2) using Sigma Scan (Imaging Technologies, Inc., Jandel Scientific, Corte Madera, CA). However, the histograms in Figure 6 were prepared by viewing HRP-stained sensory afferents under a Nikon $100 \times$ oil immersion objective at a focal plane where the majority of the sensory afferents in one-half of the neuropil were in focus. Sensory afferents in all embryonic neuropils occupied a common depth of $15 \mu \mathrm{m}$. The video image was captured through a DAJE-MTI series 68 video camera (Michigan City, IN) using Jandel video analysis software, PCVISIONPLUS ${ }^{\circledR}$ Frame Grabber version (Imaging Technologics, Inc., Jandcl Scientific). Sensory afferent projections were measured in an area of the synaptic neuropil into which the sensory afferents had entered from the peripheral nerves. During early development (stage 1 ganglia), sensory afferents enter the CNS from the median nerve; during later development (stage 2 ganglia), sensory afferents enter the CNS and from both the median and the dorsal nerves. The measured area had the following standard dimensions: the mediolateral coordinate of the area was the width of the ipsilateral neuropil; the anterior-posterior coordinate of the area had a length of $35 \mu \mathrm{m}$ (Fig. 2). Background was measured and subtracted from the image. The remaining stained area was calculated by multiplying the total area of interest by the ratio of gray pixels to total pixels in the area of interest. Only the side of each neuropil with the most extensive sensory afferent growth was measured in both the control and experimental embryos.

\section{Results}

We determined the effects of three different experimental reagents-mannose-BSA, $N$-glycanase, and Lan3-2 Fab fragments-on sensory afferent growth in cultured embryos. Different developmental stages of sensory afferent growth can be monitored in a given embryo due to the anterior to posterior developmental gradient of its 32 segmentally reiterative ganglia (Weisblat et al., 1980; Fernandez and Stent, 1982). We divided sensory afferent growth into two stages: an early developmental stage when sensory afferents grow into the CNS from only the median nerve (stage 1 ganglia), and a later developmental stage when sensory afferents grow into the CNS from both the median and dorsal nerves (stage 2 ganglia).

\section{Effects of mannose-BSA on the stabilization on sensory afferent arborization}

To strengthen the evidence that the projection of sensory afferents into the synaptic neuropil is regulated by a mannose-specific recognition, the growth of sensory afferents was examined in the presence or absence of 1-4 $\mu \mathrm{M}$ mannose-BSA (Fig. 3) in stage 1 ganglia. Sensory afferents were observed by visualizing their Lan3-2 epitope. Sensory afferents from the median nerve that had just reached the CNS at the onset of culturing (Fig. 


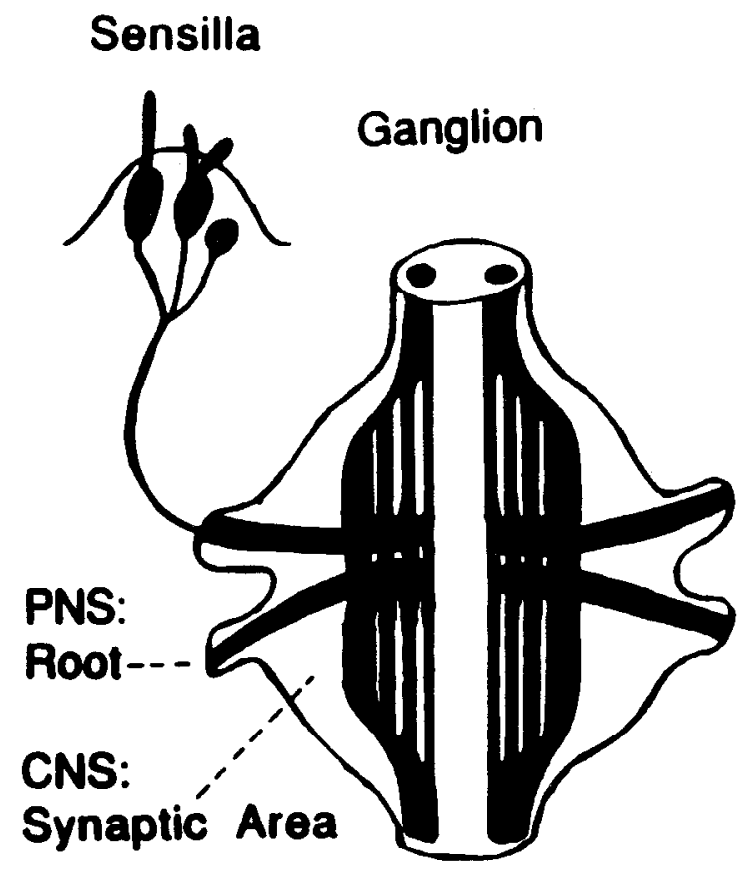

Figure 1. Schematic presentation of sensory afferent projections in an adult midbody segment. Sensory afferents of different sensory modalities (chemo-, mechano-, thermo-, and photodetectors) project axons in common, fasciculated tracts from their peripheral origin into the midbody ganglion. The different sensory modalities stereotypically project into four distinct domains within the synaptic neuropil (Peinado et al., 1987b, 1990 ) before running into the interganglionic connective. Sensory afferents express a cell-type-specific $130 \mathrm{kDa}$ surface glycoprotein, the sensory afferent gp130, which contains a mannose epitope recognized by $\mathrm{mAb}$ Lan $3-2$.

$3 A 1)$ continued to grow normally in standard culture medium. They defasciculated, projected as single axons into the synaptic area, and generated a profuse arborization (Fig. 3B1). Because of their extensive overlap, single sensory afferent axons cannot be resolved inside the arborization but only along the edges of the arborization (open arrows). In $55 \%$ of 73 ganglia exposed to $1-4 \mu \mathrm{M}$ mannose-BSA, the sensory afferents did not defasciculate but remained tightly fasciculated as they entered the $\mathrm{CNS}$ and, after bifurcating into a simple $\mathrm{T}$, elongated as a longitudinal tract in the direction of adjacent segmental ganglia (Fig. $3 C 1$ ). In the remaining $45 \%$ of the ganglia exposed to mannose-BSA, sensory afferents formed three fasciculated tracts. None of the sensory afferents underwent their normal defasciculation into the ipsilateral neuropil.

The application of mannose-BSA also affected the arborizations of median nerve sensory afferents that had formed prior to the experiment. In 50 ganglia exposed to mannose-BSA, sensory arborizations did not persist. Instead, the arborizations were replaced either by three tracts (Fig. 3C2, solid arrows; $87 \%$ of the time) or by a single longitudinal tract (Fig. $3 F$, solid arrow; $13 \%$ of the time). Typically, in the presence of mannose-BSA, single axons seen in normal or control cultured ganglia (Fig. $3 A 2, B 2$, open arrow) were absent and, instead, short stubby axonal bundles appeared (Fig. 3C2, open arrow). In contrast, sensory afferent arborizations that were formed prior to culturing (Fig. 3A2) continued to expand normally in standard culture medium (Figs. $3 B 2$ and $3 D$ ) or in the presence of $1-4 \mu \mathrm{M}$ galactose-BSA (Fig. $3 E$ ) or galactosamine-BSA.
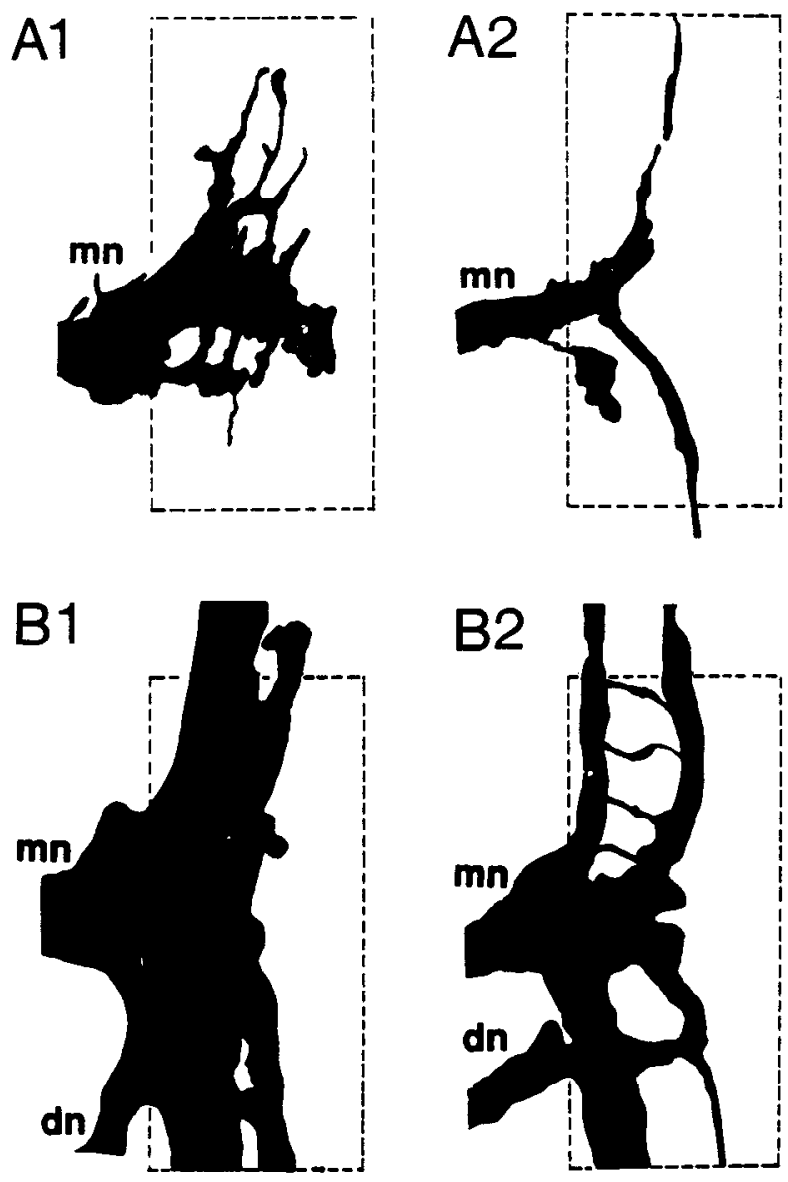

Figure 2. Measurements of sensory afferent projections by image analysis. The rectangle in drawings of sensory afferents reproduced from Figure $3 B 1(A I)$ and $3 C 1(A 2)$ and Figure $5 C 2(B I)$ and $5 D 2(B 2)$ illustrates the neuropil area in which sensory afferent projections were measured using Sigma Scan. In $A$, sensory afferents project into the neuropil via the median nerve $(m n)$, while in $B$, sensory afferents project into the neuropil via both the median and the dorsal nerves $(d n)$. The median and dorsal nerves are in the PNS, while the synaptic neuropil is in the CNS. The mediolateral coordinate of the measured area is the width of the ipsilateral neuropil; the anterior-posterior coordinate of the area had a length of $35 \mu \mathrm{m}$. In $A 2$, experimentally perturbed sensory afferents occupy $32 \%$ of the area occupied by the control cultured embryos in $A 1$. in $B 2$, experimentally perturbed sensory afferents occupy $52 \%$ of the area occupied by the control cultured embryos in $B 1$. The same area of interest defined in this figure was measured directly from embryonic ganglia through a $100 \times$ Nikon objective using Jandel video analysis software (see Fig. 6).

\section{Effects of $\mathrm{N}$-glycanase on the stabilization of sensory afferent arborization}

To test independently whether a carbohydrate domain regulates the projections of sensory afferents in the synaptic neuropil, embryos were cultured in the presence or absence of $4 \mathrm{U}$ of $\mathrm{N}$-glycanase (Fig. 4). $\mathrm{N}$-glycanase perturbations were examined in early developmental stage 1 ganglia. Previously, it had been demonstrated that $N$-glycanase cleaves the N-linked carbohydrate domain containing the Lan3-2 epitope from the sensory afferent gp130 (Bajt et al., 1990a). The sensory afferent arborization formed prior to culturing by median nerve afferents (Fig. 4AI) continued to expand in standard culture medium (data not shown). In contrast, sensory afferents in 32 ganglia exposed to $N$-glycanase formed a single anterior-posterior tract (Fig. $4 A 2 ; 62 \%$ of the time) or three tracts ( $38 \%$ of the time). 

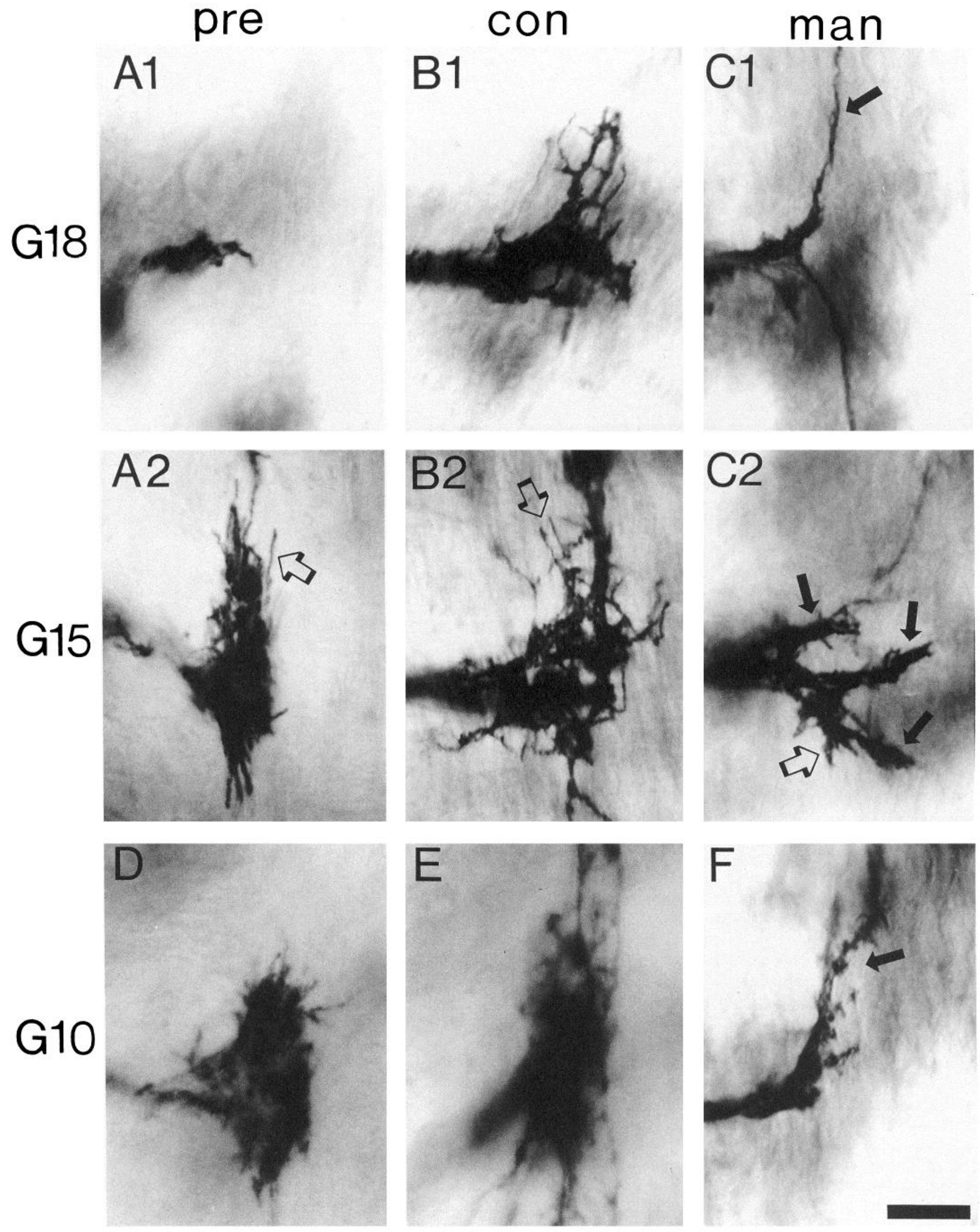

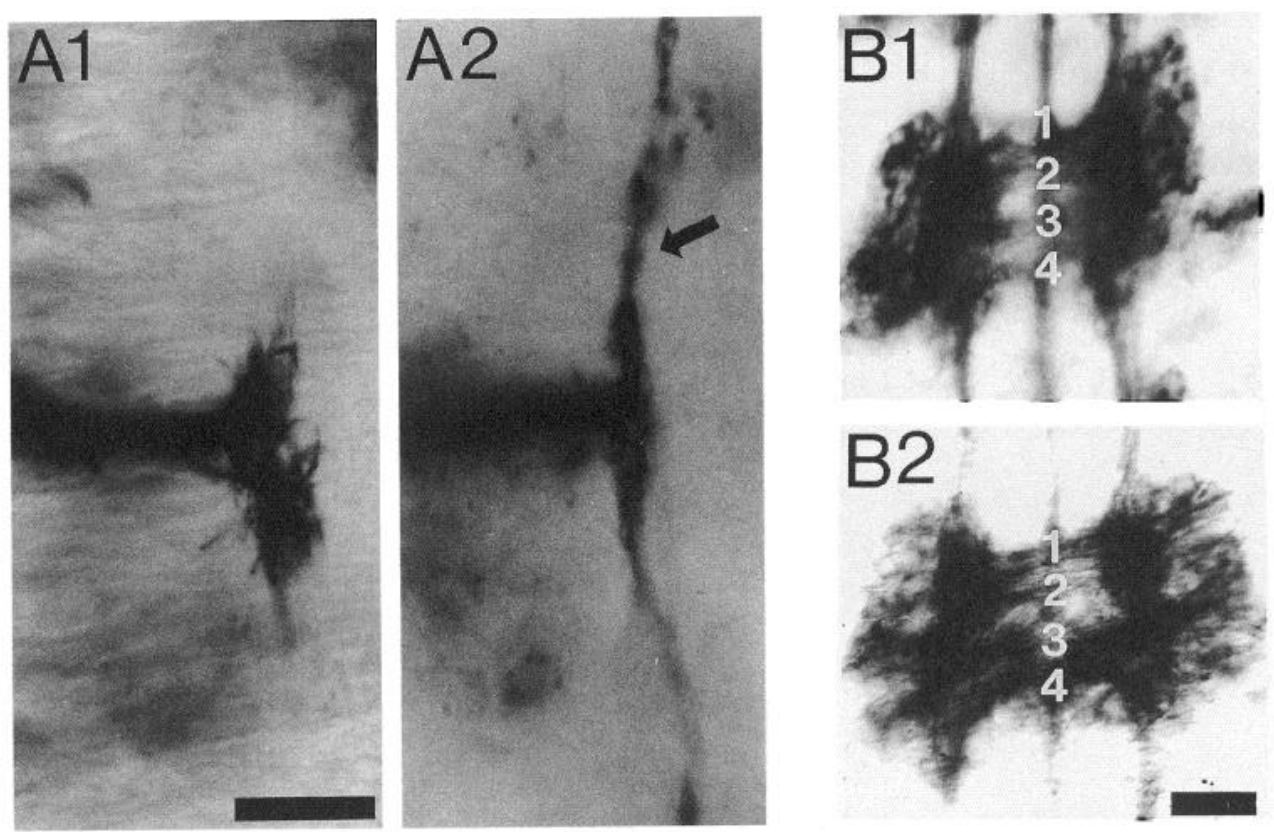

Figure 4. $\mathrm{N}$-glycanase inhibits the defasciculation of sensory afferents in the CNS. Ganglia 10 were compared in four embryos from the same cocoon that were either fixed prior to culturing or cultured for $1.5 \mathrm{~d}$ in $N$-glycanase $(4 \mathrm{U} / 300 \mu \mathrm{l})$ as described in Figure 3 . Because sensory afferents exposed to $N$-glycanase were only weakly stained using the normal procedure for visualizing the Lan 3-2 epitope, a procedure to amplify the Lan 3-2 antibody staining was used (see Materials and Methods). Prior to culturing, sensory afferents had defasciculated into a profuse arborization (A1). In a sibling embryo exposed to $N$-glycanase, sensory afferent axons grew as a single tract $(A 2$, arrow). Prior to culturing, glial processes visualized by staining with mAb Laz6-56 had formed the normal four commissures (numbered $1-4$ ) that cross the synaptic neuropil (B1). In a sibling embryo exposed to $N$-glycanase, the glial commissures also developed normally and increased in the density of glial processes (B2). Glial processes were stained with mAb Laz6-56, which reacts with a $130 \mathrm{kDa}$ glycoprotein on glial processes and produces a similar staining pattern as mAb Laz6-297 (Flaster and Zipser, 1987; Cole et al., 1989). Scale bars: $A 1$ and $A 2,10 \mu \mathrm{m} ; B 1$ and $B 2,30 \mu \mathrm{m}$.

The treatment with $N$-glycanase appeared to specifically perturb the projections of sensory afferent since the projections of glial processes in the CNS were not affected. Normally, glial processes form four major commissures that connect the right and the left neuropils (Fernandez, 1978; Cole et al., 1989). In the presence of $N$-glycanase, these glial commissures persisted and continued to proliferate (Fig. $4 B$ ). Thus, the disappearance of sensory afferent arborization was not due to a general destruction of the synaptic neuropil by $\mathrm{N}$-glycanase.

\section{Effects of mAb Lan3-2 on the stabilization of sensory afferent arborization}

Previously, we reported that the application of Lan3-2 Fab fragments inhibited the defasciculation of sensory afferents (Zipser et al., 1989). Here, we illustrate that the application of Lan3-2
Fab fragments also leads to the replacement of sensory afferent arborizations with fasciculated tracts (Fig. 5). Embryos from the same cocoon were either exposed to Lan3-2 Fab fragments or cultured under two types of control conditions. One control consisted of growing sensory afferents in standard culture medium. The other control consisted of growing sensory afferents in the presence of Laz10-1 Fab fragments. mAb Laz10-1 binds to a muscle-specific external protein (Thorey and Zipser, 1990). In the developmentally less mature ganglion 16 (stage 1 ganglion), sensory afferents have entered the CNS through the median nerve and formed an arborization prior to culturing (Fig. $5 A 1$ ). The sensory afferent arborizations continued to proliferate in embryos cultured in standard medium (Fig. $5 B 1$ ) or in the presence of $10 \mathrm{nM} \mathrm{Laz10-1} \mathrm{Fab} \mathrm{fragments} \mathrm{(Fig.} \mathrm{5Cl).} \mathrm{In} \mathrm{contrast,}$ in the presence of $10 \mathrm{nM}$ Lan3-2 Fab fragments, the sensory

Figure 3. Mannose-BSA inhibits the defasciculation of sensory afferent axons in the CNS. The siblings of a leech cocoon were divided into three categories. The preculture group (pre) was fixed prior to culturing to determine the anterior-posterior developmental gradient of sensory afferent differentiation in the 32 reiterative ganglia of the leech CNS. The control culture group (con) was cultured in standard culture medium with ( $4 \mu \mathrm{M}$ ) or without the neoglycoprotein galactose-BSA. The mannose-cultured group $(\mathrm{man})$ was grown in the presence of $1-4 \mu \mathrm{M}$ mannose-BSA. Sensory afferent growth, visualized by the Lan 3-2 epitope, was measured in ganglia that were innervated during culturing or in ganglia that were innervated $0.5 \mathrm{~d}$ prior to culturing. Sensory afferent growth is illustrated in the left half of the ganglia. Each row compares homologous ganglia from three embryos of the same cocoon. Cocoon $1: A 1, B 1$, and $C 1$ compare ganglia 18, whereas $A 2, B 2$, and $C 2$ compare the developmentally more mature ganglia 15. Ganglia 18 and 15 in each column belong to the same embryo. Prior to culturing, sensory afferent axons had not yet grown into the CNS $(A I)$. After culturing for $1.5 \mathrm{~d}$ in standard culture medium, sensory afferent axons grew into the CNS and defasciculated into an arborization (BI). In $1 \mu \mathrm{m}$ mannose-BSA, the sensory afferent axons, instead of defasciculating, elongated as a single anterior-posteriorly directed tract (C1, arrow). In the developmentally more mature ganglion 15 , sensory afferent axons had already formed an arborization prior to culturing ( $A 2)$, and after $1.5 \mathrm{~d}$ in control culture medium, the arborization widened (B2). In contrast, in $1 \mu \mathrm{M}$ mannose-BSA, the arborization collapsed into three tracts $(C 2)$. Cocoon 2: $D-F$ compare ganglia 10 of three embryos, which are of the same developmental age as ganglion 15 from the embryos in cocoon 1 . Sensory afferent axons, defasciculated prior to culturing $(D)$, continued to expand their arborization in $4 \mu \mathrm{M}$ galactose-BSA $(E)$ or elongated in a single anteriorly directed tract in $4 \mu \mathrm{M}$ mannose-BSA $(F)$. Solid arrows, major fasciculated tracts. Open arrows, single neurites or short neuritic bundles. Scale bar, $10 \mu \mathrm{m}$. 


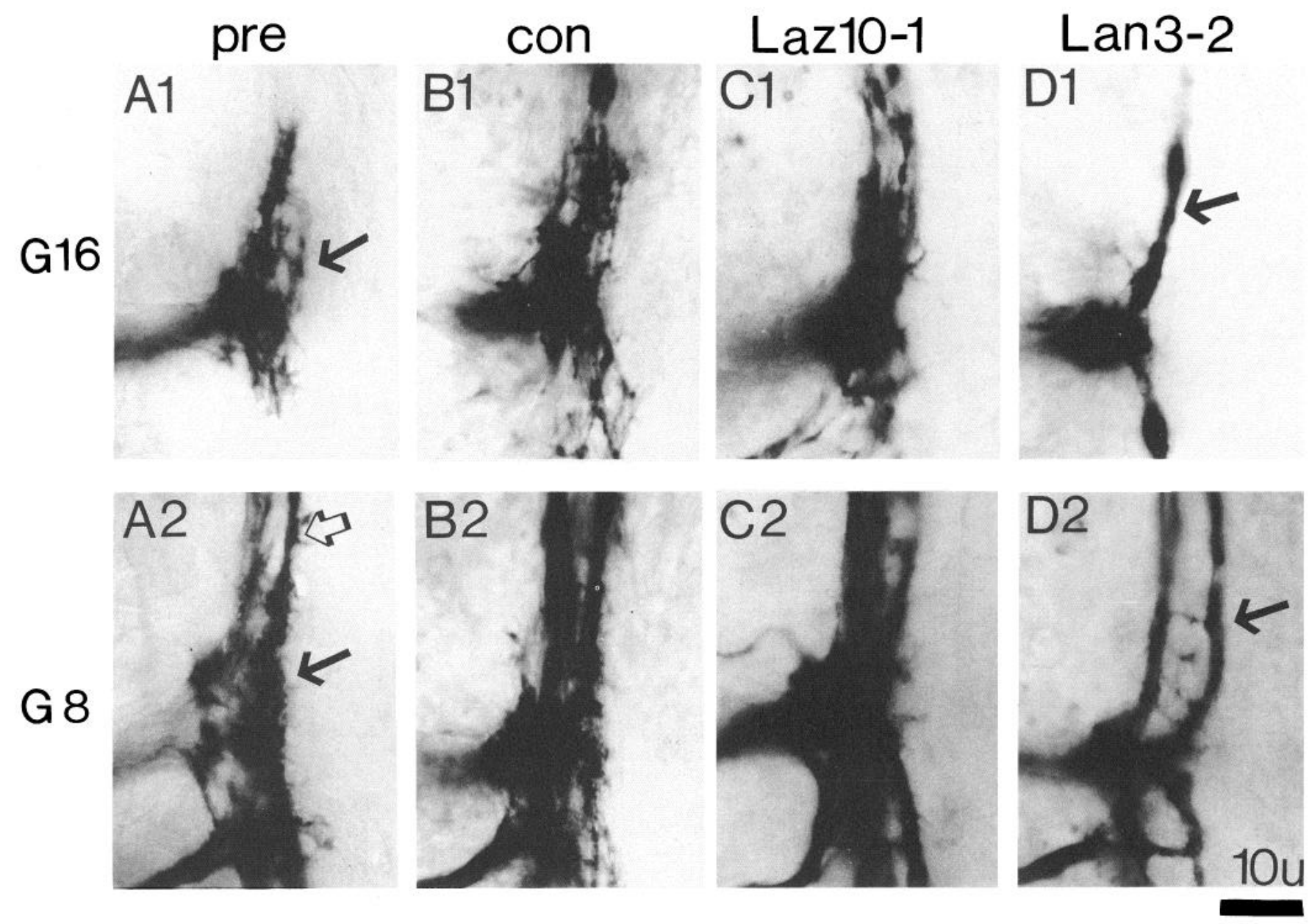

Figure 5. Lan3-2 Fab fragments inhibit the defasciculation of sensory afferent axons in the CNS. Embryos of a leech cocoon were divided into four categories. The preculture group (pre) was fixed prior to culturing; the control culture group (con) was cultured in standard culture medium; and the Laz10-1 and the Lan3-2 groups were cultured in the presence of the 10 nм Laz10-1 or Lan3-2 Fab fragments for 1.5 d. Each row compares homologous ganglia: $A 1, B 1, C 1$, and $D 1$ compare ganglia 16 , whereas $A 2, B 2, C 2$, and $D 2$ compare the developmentally more mature ganglia 8 . Each column shows ganglia from the same embryo. Sensory afferents were visualized by immunocytochemically staining their Lan3-2 epitope. Prior to culturing, sensory afferent axons had entered the CNS through the median nerve and formed an arborization (A1, arrow). In the standard culture medium $(B 1)$ or in the presence of Laz10-1 $(C 1)$, the arborization had expanded, whereas in the presence of Lan3-2 Fab fragments, the arborization was replaced with a fasciculated tract $(D 1$, arrow). In the developmentally more mature ganglia $8(A 2)$, sensory afferents entering the ganglion through the dorsal nerve contributed to the arborization (solid arrow). Two tracts were present, projecting from the arborization into the interganglion connective (open arrow). The sensory afferent arborization continued to expand in standard culture medium (B2) and in the presence of Laz10-1 Fab fragments (C2). In the presence of Lan3-2 Fab fragments, the arborization was replaced by two fasciculated tracts (D2, arrow). Scale bar, $10 \mu \mathrm{m}$.

afferent arborizations were replaced by a fasciculated tract (Fig. $5 D 1$ ). As a result, sensory afferents entering the CNS bifurcated and grew as a single anterior-posteriorly directed tract into the connective, the central axon tract linking the segmental ganglia. Thus, in Lan3-2-manipulated ganglia, there was a striking lack of arborization suggesting that the mannose-containing Lan 3-2 epitope mediates the stabilization of the defasciculated sensory afferent projections in the synaptic neuropil.

The replacement of arborizations with fasciculated tracts also occurs in the developmentally older ganglion 8 (stage 2 ganglion) of the same embryos (Fig. 5). Prior to culturing, sensory afferents entering the ganglion through the median and dorsal nerves had elaborated into a profuse arborization in the neuropil (Fig. 5A2). Two fasciculated tracts extended into the connectives from the arborization in the neuropil. The arborization continued to proliferate in the sibling embryos grown in standard culture medium (Fig. 5B2) or in the presence of Lax10-1 Fab fragments
(Fig. 5C2). The superposition of the profusely three-dimensionally projecting neurites does not allow individual neurites to be resolved in these whole-mounted ganglia. In contrast, in the presence of Lan3-2 Fab fragments, the profuse arborization in the neuropil was replaced by a pair of thick axon tracts projecting into the connectives (Fig. 5D2).

\section{Perturbation reagents reduce the area of sensory afferent projection}

We measured the effect of Lan3-2 Fab fragments and mannoseBSA at two developmental stages, in stage 1 and the older stage 2 ganglia. During stage 1, the application of mannose-BSA led to a pronounced refasciculation of sensory afferents (Fig. $6 A$ ). In control cultured ganglia, sensory afferents occupy an average of $310 \mu \mathrm{m}^{2}$. In contrast, in mannose-BSA treated ganglia, the refasciculated sensory afferents only occupied an average of 120 $\mu \mathrm{m}^{2}$. Thus, the mannose-BSA treatment led to a $61 \%$ reduction 
A

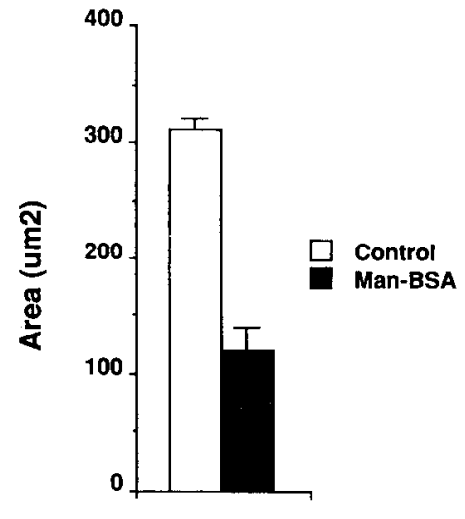

B

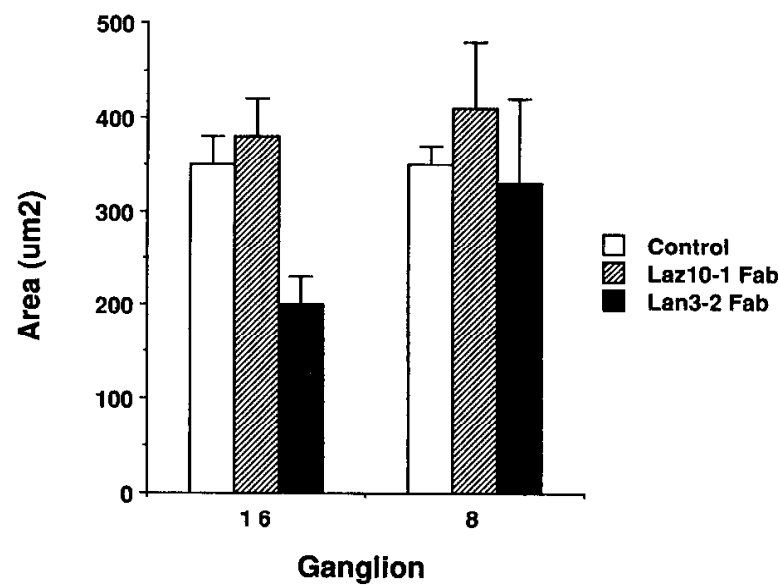

Figure 6. Effect of mannose-BSA and Lan 3-2 Fab fragments on the defasciculation of sensory afferents within the synaptic neuropil. $A$, Embryos from the same cocoon were incubated in the absence or the presence of $1 \mu \mathrm{M}$ mannose-BSA. Embryos were then fixed and stained with mAb Lan32. The average area occupied by sensory afferents was measured in the last three ganglia inncrvatcd by the sensory afferents (stage 1 ganglia) in four control cultured embryos (ranging from ganglion 15 to 18) and six mannose-BSA treated embryos (ranging from ganglion 15 to 20 ) using Jandel video analysis software. $B$, Embryos from the same cocoon were incubated in the absence or the presence of $10 \mathrm{~nm}$ Lan3-2 or $10 \mathrm{nM}$ Laz10-1 Fab fragments. Embryos were then fixed and stained with $\mathrm{mAb}$ Lan3-2. Ganglia 16 represent stage 1 ganglia, whereas ganglia 8 represent the developmentally older stage 2 ganglia. The average area occupied by sensory afferents was measured in ganglia 8 and 16 of control $(n=3)$, Laz10-1 $(n=3)$, and Lan3-2 $(n=4)$ embryos using Jandel video analysis software. Error bars represent SD.

in area. Sensory afferents exposed to Lan3-2 Fab fragments in stage 1 ganglia also demonstrated refasciculation (Fig. 6B). Control cultured sensory afferents grown either in standard culture medium or in the presence of the muscle-specific Laz10-1 Fab fragments occupied an average of 350 or $380 \mu \mathrm{m}^{2}$, respectively. In contrast, sensory afferents grown in the presence of Lan 3-2 Fab fragments only occupied $200 \mu \mathrm{m}^{2}$. Thus, the refasciculation of sensory afferents led to a $43 \%$ reduction in neuropil area occupied by the sensory afferents.

In the developmentally older stage 2 ganglia, the image analysis used here no longer detected a statistically significant effect (Fig. 6B). The amount of area occupied by sensory afferents in control ganglia was the same as seen in stage 1 ganglia because the sensory afferents project over a diameter of $15 \mu \mathrm{m}$ in the ipsilateral neuropil. There was, however, no longer a measurably significant reduction in sensory afferent area when stage 2 ganglia were treated with Lan3-2 Fab fragments, yet all visually inspected ganglia had a clearly enhanced refasciculation. Instead of the profuse arborization, thicker tracts were commonly seen (Fig. 5D2). The method we used apparently was not sensitive enough to measure the visually observed refasciculation in terms of reduction in area occupied by sensory afferents in stage 2 ganglia.

\section{Discussion}

Indirect evidence for carbohydrate interactions during neuronal pattern formation has come from studying the spatiotemporal expression of cell surface carbohydrates and carbohydratc-binding proteins using antibodies or lectins. Carbohydrates and carbohydrate-binding proteins were found to label functional classes of neurons (Bajt et al., 1990a; Hynes et al., 1990; S. A. Scott et al., 1990; and as reviewed in Jessell et al., 1990), growth cones (Pfenninger et al., 1984), and synaptic sites (Dontenwill et al., 1985; Scott et al., 1988) and to distinguish between neural and non-neural cells (Snow et al., 1987). The expression and distribution of oligosaccharides has been shown to change when syn- aptic transmission is blocked or when neurons and muscles are denervated (Dontenwill et al., 1985; Naegele and Katz, 1990; L. J. C. Scott et al., 1990). Direct evidence for oligosaccharides mediating neuronal migration and axonal growth has been obtained by experimentally manipulating the interactions betwecn carbohydrates and their carbohydrate-binding proteins in dissociated cells (Runyan et al., 1988) or in brain explants (Antonicek et al., 1987). We are directly investigating the biological role of carbohydrate recognition in the leech embryonic nervous system by experimentally manipulating neurons in their normal cellular and molecular environment.

\section{Defasciculation versus fasciculation}

Since sensory afferents are uniquely labeled with a Lan3-2 reactive $130 \mathrm{kDa}$ surface protein, it seemed plausible that some aspect of sensory afferent growth is mediated by this surface protein. Initially, the fasciculated projections of the sensory afferents were studied in peripheral and central nerves (Flaster et al., 1983; McKay et al., 1983; Peinado et al., 1987b). A subsequent study demonstrated that following a nerve lesion, regenerating sensory afferents realigned with previously formed fascicles of sensory afferents (Peinado et al., 1987a). The idea then arose that the fasciculated growth of sensory afferents might be mediated by the sensory afferent gp 130. Other surface molecules such as N-CAM and L1 that are expressed by many functional classes of neurons have been demonstrated, using antibody perturbations, to mediate axonal fasciculation (reviewed in Rutishauser and Jessell, 1988), yet, as discussed below, we have shown that the Lan 3-2 epitope of sensory afferent gp130 mediates the defasciculation of sensory afferent axons.

\section{Three lines of evidence for mannose-specific recognition in the synaptic neuropil}

We have presented three lines of direct experimental evidence indicating that a mannose-specific recognition mediates neuronal pattern formation in the leech CNS. During leech neuronal 
development, the defasciculation in the CNS of bundled sensory afferent axons is a necessary step in the formation of the adult CNS pattern. Using antibody perturbation, we obtained our first line of evidence that this defasciculation is mediated by a mannose-specific recognition involving the Lan3-2 epitope of sensory afferents. The binding of Lan3-2 Fab fragments to a mannose-containing epitope located on the sensory afferent gp 130 inhibited the defasciculation of sensory afferents (Zipser et al., 1989). This Lan3-2 antibody perturbation effect, however, does not necessarily involve the mannose-containing epitope since the binding of Lan3-2 Fab fragments to its epitope may have sterically obstructed adjacent functional domains. Therefore, as a second line of experimental evidence, the involvement of $\mathrm{N}$-linked carbohydrate domains in axonal defasciculation, was demonstrated by treating the cultured embryos with $N$-glycanase.

Previously, using $N$-glycanasc it was shown that the mannosecontaining Lan3-2 epitope is located on an N-linked carbohydrate domain (Bajt et al., 1990a). Cleaving N-linked carbohydrate domains also prevented the defasciculation of sensory afferents. Sensory afferents may be only partially deglycosylated because it was possible to observe the sensory afferents treated with $N$-glycanase by using a procedure to amplify the standard Lan3-2 antibody staining method. This suggests that the normal defasciculations and arborizations of the sensory afferents require a critical level of the Lan3-2 epitope. Alternatively, all of the sensory afferents may have been denuded of the Lan3-2 epitope at the membrane surface. Because antibody staining was carried out in the presence of detergent, perhaps the sensory afferents still could be visualized due to the presence of internal Lan3-2 epitopes.

The removal of $\mathrm{N}$-linked glycans by $N$-glycanase may have made the protein cores of glycoproteins on glial and ncrve cclls more susceptible to proteolytic action, thereby altering sensory afferent development. The normal appearance of glial processes in ganglia treated with $N$-glycanase, however, suggests that the replacement of sensory afferent arborization with fasciculated tracts was not due to a nonspecific proteolytic destruction of the neuropil organization.

The third line of experimental evidence for a mannose-specific recognition was obtained by exposing growing sensory afferents to mannose-BSA. Previously, it had been demonstrated that the Lan3-2 epitope contains mannose by blocking the binding of mAb Lan3-2 to its epitope with 100-500 mM $\alpha$-methylmannoside (McKay et al., 1983; Bajt et al., 1990a). Because sensory afferent growth was not perturbed by up to $50 \mathrm{~mm}$ monosaccharide $\alpha$-methyl-mannoside (B. Zipser and R. N. Cole, unpublished observations), we instead used mannose-BSA as multivalent soluble inhibitor. The application of micromolar concentrations of mannose-BSA mimicked the effects obtained by exposing sensory afferents to Lan3-2 Fab fragments or $N$-glycanase. Presumably, the mannose-BSA competed with the mannose-containing Lan3-2 epitope for a putative mannose-binding protein.

These experiments predict the presence of a mannose-binding protein in the synaptic neuropil. The neuropil is occupied by processes of ganglionic cells such as the bipolar cells (Stewart et al., 1987) and glial cells (Cole et al., 1989) before the appearance of sensory afferents. The putative mannose-binding protein could be made by glial processes in the synaptic neuropil or, alternatively, by neurons that are postsynaptic to the sensory afferents. Sensory afferents are within easy reach of their postsynaptic neurons once they have arrived in the CNS. The ip- silateral neuropil has a mediolateral dimension of about $15 \mu \mathrm{m}$, which is matched by the approximately $15-\mu \mathrm{m}$-long excursions (Zipser et al., 1989) made of sensory afferents. Thus, the initially small size of the neuropil and the natural propensity of the sensory afferent axons for short independent excursions obviates the need for additional pathfinding clues to lead sensory afferents to their postsynaptic partners.

The possibility must be considered that each type of perturbation experiment inhibits the defasciculation of sensory afferents by interfering with a separate molecular mechanism. The results from each perturbation experiment, however, indicate that the molecular mechanisms of sensory afferent defasciculation at least are related. The $N$-glycanase perturbation, cleaving $\mathrm{N}$-linked carbohydrate domains potentially on the surface of all cells, implicates a carbohydrate interaction in the defasciculation. The mannose-BSA perturbation, blocking mannose rccognition/adhesion events, focuses on a specific carbohydratc interaction, mannose, mediating the defasciculation. The Lan3-2 perturbation implicates the mannose-containing carbohydrate domain of the sensory afferent glycoprotein in mediating the defasciculation. Together, these three independent experimental perturbations and the characteristics of the Lan3-2 epitope provide strong evidence that the defasciculation is mediated by the Lan3-2 epitope (Fig. 7).

\section{Stabilization of sensory afferent arborization as mediated by a mannose-specific recognition}

As sensory afferents differentiate, they grow into the CNS as a fasciculated tract. Upon entering the CNS, the sensory afferents defasciculate and form an arborization in the synaptic neuropil. With ongoing development, new sensory afferents grow into the CNS and contribute to the arborization. Not only the initial defasciculation of sensory afferents but also their continuing arborization in the neuropil appear to be dependent on the mannose-specific recognition. When the interactions of the Lan3-2 epitope are manipulated, sensory afferent arborizations are replaced by fasciculated tracts. This occurs in synaptic neuropils that are solely innervated by sensory afferents via the median nerve. Presumably, the defasciculated sensory afferents are retracted from their various neuropil locations and reassembled into the fasciculated tracts. The inhibition of defasciculation causes a $43-61 \%$ reduction in neuropil area that is occupied by sensory afferents. The defasciculation is inhibited to a variable degree in developmentally older ganglia in which sensory afferents from both the median and dorsal nerves contribute to the neuropil arborization. The variation in inhibition of defasciculation in these developmentally older ganglia correlates with a change in surface proteins that express the Lan 3-2 epitope. Early differentiating scnsory affercnt glycoproteins express the mannose-containing Lan3-2 epitope only on sensory afferent gp 130, while later in development and in adulthood, the Lan3-2 epitope is also expressed on sensory afferents gp 103 and gp95 (McGlade-McCulloh et al., 1990). The sensory afferent gp130 behaves as a peripheral membrane protein, while the sensory afferents gp 103 and gp 95 behave as integral membrane proteins (Bajt et al., 1990a). Displaying the Lan3-2 epitope on peripheral membrane proteins may allow a sensory afferent the flexibility to extend easily and to retract its axon while it is searching for its postsynaptic targets. The appearance of the Lan3-2 epitope on integral membrane proteins later during sensory afferent differentiation may contribute to stabilizing sensory afferent nerve terminals, thereby reducing the degree to which sensory afferents refasciculate. It is possible that sensory afferent stabilization 


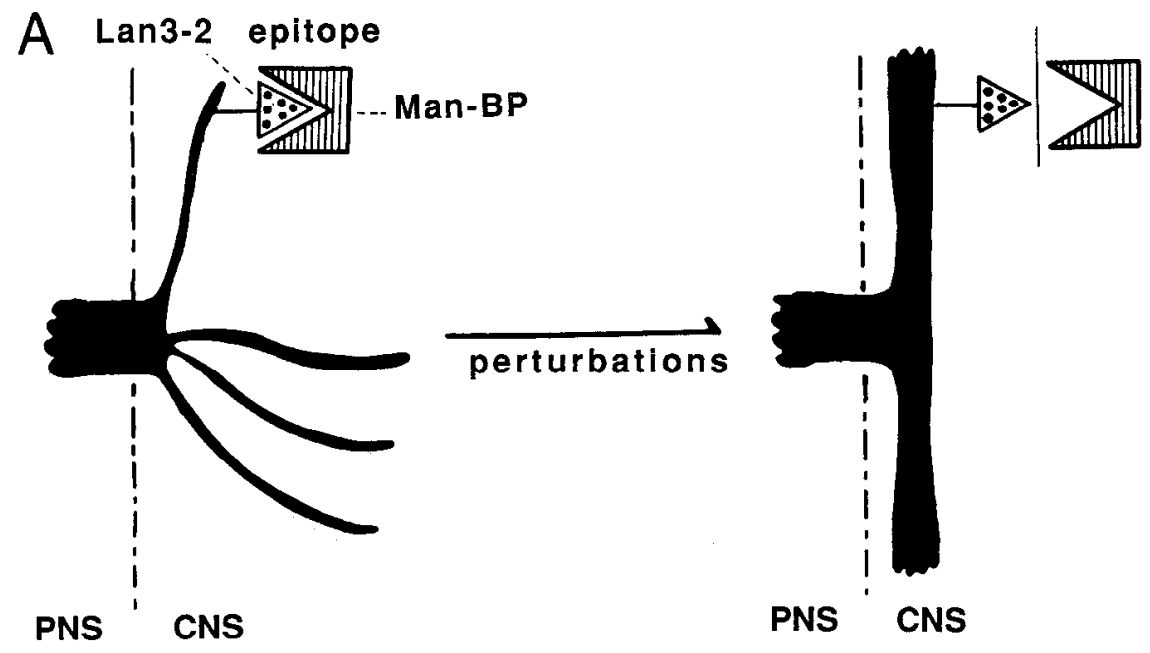

Figure 7. Hypothesis of carbohydrate recognition mediating the defasciculation of sensory afferents into the synaptic neuropil. $A$, During development, sensory afferent axons grow as a fasciculated bundle through a peripheral nerve $(P N S)$ into a central ganglion (CNS), where they defasciculate, projecting as single axons through the synaptic neuropil. Even though the mannose-containing Lan3-2 epitope is evenly distributed on the sensory afferent surface, the defasciculation event is restricted to the synaptic neuropil. Our experimental evidence is consistent with a mannose-binding protein (Man-BP) being present in the synaptic neuropil. Recognition/adhesion between the Lan3-2 epitope of sensory afferents and the $M a n-B P$ in the synaptic neuropil results in the region-specific defasciculation. Perturbations of this mannose-specific recognition inhibit the defasciculation, and, instead, sensory afferents continue to grow through the CNS as a bifurcated fasciculated tract, appearing as a T. $B$, The normal recognition between the Lan3-2 epitope and the Man-BP is inhibited in the presence of (1) Lan3-2 $F a b$ fragments; (2) $N$-glycanase, a glycosidase; and (3) mannose-BSA (man$B S A$ ), a neoglycoprotein. The $\operatorname{Lan} 3-2$ $F a b$ fragments bind to and mask the mannose-containing Lan3-2 epitope, $N$-glycanase cleaves $\mathrm{N}$-linked carbohydrate domains removing the Lan 3-2 epitope, and man-BSA competes with the Lan3-2 epitope for the mannosebinding protein.

results from cytoskclctal protcins in scnsory afferent nerve terminals being linked to the Lan3-2 epitope on sensory afferents via gp95 and gp 103, which are bound to an external mannosebinding protein.

\section{Axonal fasciculation in peripheral and central axon tracts}

Sensory afferents grow as fasciculated tracts through peripheral and central nerves. The molecular mechanisms that lead to sensory afferent fasciculation are not known. We have presented evidence that the mannose-containing Lan3-2 epitope of the sensory afferent gp130 is not involved in fasciculation. However, this does not rule out that other domains of the sensory afferent $\mathrm{gp} 130$, for example the protein core, may mediate fasciculation. Alternatively, the fasciculation could be mediated by three other carbohydrate epitopes expressed by subsets of sensory afferents or by an unrelated surface protein, such as the HNK-1 epitope (Bajt et al., 1990a,b).

\section{References}

Antonicek H, Persohn E, Schachner M (1987) Biochemical and functional characterization of a novel neuron-glia adhesion molecule that is involved in neuronal migration. J Cell Biol 104:1587-1595.

Bajt ML, Cole RN, Zipser B (1990a) The specificity of the 130-kD leech sensory afferent proteins is encoded by their carbohydrate epitopes. J Neurochem 55:2117-2125.

Bajt ML, Schmitz B, Schachner M, Zipser B (1990b) Carbohydrate epitopes involved in neural cell recognition are conserved between vertebrates and leech. J Neurosci Res 27:276-285.

Bradford MM (1976) A rapid and sensitive method for the quantitation of microgram quantities of protein utilizing the principle of protein-dye binding. Anal Biochem 12:248-254.

Cole RN, Morell RJ, Zipser B (1989) Glial processes, identified through their glial-specific $130 \mathrm{kD}$ surface glycoprotein, are juxtaposed to sites of neurogenesis in the leech germinal plate. Glia 2:446-457.

Dontenwill M, Roussel G, Zanetta JP (1985) Immunohistochemical localization of a lectin-like molecule, $\mathrm{R}$, during the postnatal development of the rat cerebellum. Dev Brain Res 17:245-252.

Fernande7. J (1978) Structure of the leech nerve cord: distribution of neurons and organization of fiber pathways. J Comp Neurol 180:165191.

Fernandez J, Stent GS (1982) Embryonic development of the hirudinid leech Hirudo medicinalis: structure, development and segmentation of the germinal plate. J Embryol Exp Morphol 72:71-96.

Flaster MS, Zipser B (1987) The macroglial cells of the leech are molecularly heterogeneous. J Neurosci Res 17:176-183.

Flaster MS, Schley C, Zipser B (1983) Generating monoclonal antibodies against excised gel bands to correlate immunocytochemical and biochemical data. Brain Res 277:196-199.

Hynes MA, Gitt M, Barondes SH, Jessell TM, Buck LB (1990) Selective expression of an endogenous lactose-binding lectin gene in subsets of central and peripheral neurons. J Neurosci 10:1004-1013.

Jessell TM, Hynes MA, Dodd J (1990) Carbohydrates and carbohydrate-binding proteins in the nervous system. Annu Rev Neurosci 13:227-255.

McGlade-McCulloh E, Muller KJ, Zipser B (1990) Expression of surface glycoproteins in leech neural development. J Comp Neurol 299: 123-131.

McKay RDG, Hockfield S, Johansen I, Thompson I, Frederiksen K 
(1983) Surface molcculcs identify groups of growing axons. Scicnce 222:678-684.

Mishell BB, Shiigi SM (1980) Selected methods in cellular immunology. New York: Freeman.

Naegele JR, Katz LC (1990) Cell surface molecules containing $N$-acetylgalactosamine are associated with basket cells and neuroglialform cells in cat visual cortex. J Neurosci 10:540-557.

Ofek I, Sharon N (1988) Lectinophagocytosis: a molecular mechanism of recognition between cell surface sugars and lectins in the phagocytosis of bacteria. Infec Immun 56:539-547.

Peinado A, Zipser B, Macagno E (1987a) Regeneration of afferent axons into discrete tracts within peripheral nerves in the leech. Brain Res 410:330-334.

Peinado A, Macagno ER, Zipser B (1987b) A group of related surface glycoproteins distinguish sets and subsets of sensory afferents in the leech nervous system. Brain Res 410:335-339.

Peinado A, Zipser B, Macagno E (1990) Segregation of afferent projections in the central nervous system of the leech Hirudo medicinalis. J Comp Neurol 301:232-242.

Pfenninger KH, Maylié-Pfenninger MF, Friedman LB, Simkowitz P (1984) Lectin labeling of sprouting neurons. III. Type-specific glycoconjugates on growth cones of different origin. Dev Biol 106:97108.

Runyan RB, Versalovic J, Shur BD (1988) Functionally distinct laminin receptors mediate cell adhesion and spreading: the requiremen for surface galactosyltransferase in cell spreading. $\mathbf{J}$ Cell Biol 107: 1863-1871.

Rutishauser U, Jessell TM (1988) Cell adhesion molecules in vertebrate neural development. Physiol Rev 68:819-856.

Scott LJC, Francis B, Sanes JR (1988) A synapse-specific carbohydrate at the neuromuscular junction: association with both acetylcholinesterase and glycolipid. J Neurosci 8:932-944.

Scott LJC, Balsamo J, Sanes JR, Lilien J (1990) Synaptic localization and neural regulation of an $N$-acetylgalactosaminyl transferase in skeletal muscle. J Neurosci 10:346-350.

Scott SA, Patel N, Levine JM (1990) Lectin binding identifies a subpopulation of neurons in chick dorsal root ganglia. J Neurosci 10: 336-345.

Shur B (1989) Expression and function of cell surface galactosyltransferase. Biochim Biophys Acta 988:389-409.

Snow PM, Patel NH, Harrelson AL, Goodman SC (1987) Neuralspecific carbohydrate moiety shared by many surface glycoproteins in Drosophila and grasshopper embryos. J Neurosci 7:4137-4144.

Stewart RR, Gao W, Peinado A, Zipser B, Macagno ER (1987) Cell death during gangliogenesis in the leech: bipolar cells appear and then degenerate in all ganglia. J Neurosci 7:1919-1927.

Thorey IS, Zipser B (1991) The segmentation of the leech nervous system is prefigured by myogenic cells at the embryonic midline expressing a muscle-specific matrix protein. J Neurosci 11:1786-1799.

Weisblat DA, Harper G, Stent GS, Sawyer RT (1980) Embryonic cell lineages in the nervous system of the glossiphoniid leech Helobdella triserialis. Dev Biol 76:58-78.

Yednock TA, Rosen SD (1989) Lymphocyte homing. Adv Immunol 44:313-378.

Zipser B, McKay R (1981) Monoclonal antibodies distinguish identifiable neurons in the leech. Nature 289:549-554.

Zipser B, Morell R, Bajt ML (1989) Defasciculation as a neuronal pathfinding strategy: involvement of a specific glycoprotein. Neuron $3: 621-630$. 